\title{
Postoperative satisfaction following lumbar spinal fusion surgery: patient expectation versus actuality
}

\author{
Joon S. Yoo, BA, Dil V. Patel, BS, Benjamin C. Mayo, MD, Dustin H. Massel, MD, \\ Sailee S. Karmarkar, BS, Eric H. Lamoutte, BS, and Kern Singh, MD \\ Department of Orthopaedic Surgery, Rush University Medical Center, Chicago, Illinois
}

\begin{abstract}
OBJECTIVE Due to the reported benefits associated with minimally invasive spine surgery (MIS), patients seeking out minimally invasive surgery may have higher expectations regarding their outcomes. In this study the authors aimed to assess the effects of preoperative expectations and postoperative outcome actuality, and the difference between the two, on postoperative satisfaction following MIS for lumbar fusion procedures.
\end{abstract}

METHODS Patients scheduled for either a 1- or 2-level lumbar fusion MIS were administered confidential surveys preoperatively and at 6 months postoperatively. The surveys administered preoperatively consisted of 2 parts: preoperative patient-reported outcomes (PROs), including the Oswestry Disability Index (ODI), visual analog scale (VAS) back pain, and VAS leg pain, and expected postoperative PROs. The surveys administered 6 months postoperatively consisted of 2 parts: postoperative PROs and satisfaction. Preoperative symptoms, expected postoperative symptoms, and actual postoperative symptoms were compared using paired t-tests. Pearson correlation was used to compare the association between 1) postoperative change in PROs and satisfaction, 2) expectation and satisfaction, 3) expectation-actuality discrepancy and satisfaction, and 4) actuality and satisfaction.

RESULTS In total, 101 patients completed all surveys. Patients expected to improve in all PROs from baseline, except for ODI personal care, in which they expected to get worse after surgery. In actuality, patients improved in all PROs from baseline, except for ODI personal care, in which they did not demonstrate improvement or worsening. Patients did not surpass any expectations regarding PRO improvement. The association between patient satisfaction and postoperative change was strong for the VAS back pain score, while ODI and VAS leg pain scores showed moderate correlations. Preoperative expectation and postoperative satisfaction demonstrated weak to moderate correlations for all outcome measures. All 3 PROs demonstrated moderate correlation between patient satisfaction and the expectation-actuality discrepancy. All 3 PROs demonstrated strong correlations between satisfaction and actual postoperative outcomes, with ODI having the strongest correlation.

CONCLUSIONS In this observational study, the authors determined that the actual postoperative results following surgery were strongly correlated with patient satisfaction, while the patients' expectation, the expectation-actuality discrepancy, and the postoperative improvement did not demonstrate strong correlations for all patient-reported outcome measures utilized in this study. The investigation results suggest that the most important indicator of how satisfied patients feel following surgery may be the actual outcome itself, rather than the preoperative expectation or the degree to which the expected result was met.

https://thejns.org/doi/abs/10.3171/2019.5.SPINE19213

KEYWORDS lumbar fusion; patient expectations; patient-reported outcomes; Oswestry Disability Index; visual analog scale

$\mathrm{P}$ ATIENT satisfaction is an ever-increasing priority in quality assessment in orthopedic and spine surgery. The expectations patients have regarding postoperative outcomes may influence their satisfaction with the procedure, and ultimately, rapport with the treating surgeon and institution. In the current healthcare climate where an emphasis has been placed on value-based care, it is imperative to investigate both psychological and biological factors that may influence patient satisfaction with common procedures.

Prior studies have investigated the relationship between expectations and satisfaction for patients undergoing spine

ABBREVIATIONS MIS = minimally invasive spine surgery; ODI = Oswestry Disability Index; PRO = patient-reported outcome; VAS = visual analog scale.

SUBMITTED February 25, 2019. ACCEPTED May 3, 2019.

INCLUDE WHEN CITING Published online July 26, 2019; DOI: 10.3171/2019.5.SPINE19213. 
surgery. While some studies have demonstrated a positive association between preoperative expectation and postoperative satisfaction, 1,4,5,8-11,13-15,19 others have found a negative association. ${ }^{6,7}$ Additionally, several studies have posited that the most important predictor of patient satisfaction is the expectation-actuality discrepancy, or the difference between preoperative expectations and the actual postoperative outcomes following surgery. ${ }^{12,16,18}$ However, a majority of the studies reporting these findings have examined patients undergoing open lumbar surgery and not minimally invasive spine surgery (MIS).

Due to the reported benefits associated with MIS, patients seeking out minimally invasive lumbar surgery may have higher expectations regarding their outcomes. As such, the difference between expectations and actuality may play a larger role in satisfaction following MIS compared to open surgeries. The purpose of this study was to assess the effect of preoperative expectations, postoperative outcome actuality, and the difference between the two on postoperative satisfaction following MIS lumbar fusion procedures.

\section{Methods \\ Patient Population}

Following Institutional Review Board approval (ORA no. 15101503), patients scheduled for either a 1- or 2-level MIS lumbar fusion surgery for degenerative pathology were administered a pair of confidential surveys from October 2016 to July 2018. All patients in our study were treated by a single surgeon at a single academic medical center. Patients were included if they were fluent in English and older than 18 years. Patients were excluded if they were unable to answer questions associated with the study, had had any spinal procedures within the past year, or had nondegenerative indications for surgery (i.e., active infection, malignancy, or trauma).

\section{Patient Survey}

All patients who consented to participate in our study received an electronic version of the surveys at the preoperative appointment and again at the 6-month postoperative appointment. Patient counseling took place during the same preoperative appointment in which surgery was offered. The surveys were administered after patient counseling occurred. The senior surgeon provided patient counseling, whereas advanced practice providers, fellows, residents, and medical students were not involved in any counseling. In addition to the surveys, data were collected regarding patient demographic characteristics, including sex, age, ethnicity, and insurance status.

The surveys administered at the preoperative appointment consisted of 2 parts: preoperative patient-reported outcomes (PROs) and expected postoperative PROs. The preoperative PROs were determined on the basis of patient survey scores for the Oswestry Disability Index (ODI), visual analog scale (VAS) back pain, and VAS leg pain. In conjunction with completing the first set of PRO surveys based on the current symptoms they were having at the preoperative time point, patients were also asked to respond to the same questions by indicating the outcomes they were expecting to attain following their MIS lumbar fusion procedure.

The surveys administered at the 6-month postoperative appointment consisted of 2 parts: postoperative PROs and satisfaction. The postoperative PROs consisted of the same questionnaires as the preoperative surveys (i.e., ODI, VAS back pain, and VAS leg pain). The patients were then asked to rate their overall satisfaction with each of the PROs with the use of another VAS scoring system, with a numerical scale from 0 points (the least satisfactory score) to 10 points (the most satisfactory score). The VAS scoring system was utilized to assess patient satisfaction in our study based on prior literature that determined this assessment method to be less vulnerable to bias from confounding factors and the ceiling effect in comparison to other psychometric measurement instruments such as the Likert scale. ${ }^{17}$

\section{Statistical Analysis}

Statistical analysis was performed using Stata/MP 13.0 (StataCorp LP). Patient demographics were analyzed with descriptive statistics. Preoperative symptoms, expected postoperative symptoms, and actual postoperative symptoms were compared using paired t-tests. Pearson correlation was used to compare the associations between 1) postoperative change in PROs and satisfaction, 2) expectation and satisfaction, 3) expectation-actuality discrepancy and satisfaction, and 4) actuality and satisfaction. The level of significance was set as $\mathrm{p}<0.05$.

\section{Results}

A total of 101 patients completed all surveys and were included in the analysis. The average age was 57 years and $57.4 \%$ of patients $(n=58)$ were males. Most patients were classified as Caucasians $(79.2 \%, \mathrm{n}=80)$ and had private insurance $(62.4 \%, \mathrm{n}=63)$. Demographics and baseline characteristics are further characterized in Table 1.

Tables 2 and 3 compare preoperative, expected, and postoperative PROs. When comparing preoperative PROs to expected postoperative PROs, patients expected to improve in all PROs from baseline, except for ODI personal care, in which they expected to get worse after surgery. When comparing preoperative PROs to actual postoperative PROs, patients improved from baseline in all PROs, except for ODI personal care, in which they did not demonstrate improvement or worsening. When comparing expected postoperative PROs to actual postoperative PROs, patients did not surpass any expectations regarding PRO improvement. Patients met expectations in ODI pain intensity, personal care, lifting, and walking; however, they failed to meet expectations regarding other ODI subcomponents as well as VAS back and leg pain. Table 4 lists the outcomes of these comparisons.

Table 5 displays correlations of preoperative, expected, and postoperative PROs and satisfaction. When analyzing the association between patient satisfaction and change in postoperative PROs from preoperative baseline, VAS back pain had a strong correlation while ODI and VAS leg pain had moderate correlations. When analyzing the association between preoperative expectation and postoperative satisfaction, there were weak to moderate correlations for 
TABLE 1. Baseline characteristics

\begin{tabular}{cc}
\hline Characteristic & Patients, \% (no.) \\
\hline Sex & \\
\hline Female & $42.6 \%(43)$ \\
\hline Male & $57.4 \%(58)$ \\
\hline Age, yrs & $5 \%(5)$ \\
\hline $30-39$ & $14.9 \%(15)$ \\
\hline $40-49$ & $34.7 \%(35)$ \\
\hline $50-59$ & $34.7 \%(35)$ \\
\hline $60-69$ & $10.9 \%(11)$ \\
\hline$\geq 70$ & \\
\hline Ethnicity & $79.2 \%(80)$ \\
\hline Caucasian & $5.9 \%(6)$ \\
\hline African American & $11.9 \%(12)$ \\
\hline Hispanic & $3.0 \%(3)$ \\
\hline Asian & \\
\hline Insurance & $11.9 \%(12)$ \\
\hline Medicare/Medicaid & $25.7 \%(26)$ \\
\hline Workers' compensation & $62.4 \%(63)$ \\
\hline Private &
\end{tabular}

all outcome measures. When analyzing the association between patient satisfaction and the expectation-actuality discrepancy, all 3 PROs had moderate correlations. When analyzing the association between patient satisfaction and actual postoperative PROs, there were strong correlations in each of the 3 PROs, with ODI scores having the strongest correlation (Figs. 1-3).

\section{Discussion}

The quality of healthcare delivered to patients is increasingly being assessed through patient-centered metrics, such as self-reported satisfaction and perception of outcomes. With the establishment of organizations such as the Patient Centered Outcomes Research Institute, the current medical climate has been trending toward improving care by obtaining a better understanding of factors that can prognosticate clinical outcomes. ${ }^{3}$ Expectations that patients harbor at baseline have been shown to influence patient-reported outcomes after treatment or surgical intervention. ${ }^{2,4}$ Few prior investigations have examined the relationship between preoperative expectation and postop- erative satisfaction for patients undergoing MIS. As such, we conducted an observational study in order to explore the impact on postoperative satisfaction of preoperative expectations, postoperative outcome actuality, and the difference between the two.

The results of our study demonstrate that patients expected to have significant improvements from their preoperative baseline in their postoperative outcomes. Patients expected greater than 50\% improvement from baseline as reflected in scores for ODI, VAS back pain, and VAS leg pain. Furthermore, postoperative PROs, termed patient actuality, were determined to be significantly improved from baseline for all outcome measures, with patients experiencing greater than $40 \%$ improvement from the preoperative time point. However, the actual postoperative outcomes were determined to be significantly worse than the expected results for ODI and VAS back and leg pain. Therefore, while patients made significant improvements following MIS lumbar fusion, their actual postoperative outcomes did not meet their preoperative expectations.

Analysis of the ODI and VAS back and leg pain scores, as well as subanalysis of the ODI components, revealed interesting results. When comparing patient expectations to preoperative baseline scores, patients expected significant improvements in ODI and VAS back and VAS leg pain scores and in all of the ODI subcomponents except for personal care, for which patients expected significantly worse outcomes. One plausible explanation is that the personal care component of the ODI details activities of daily living such as washing and dressing, with which patients may have expected to need significant assistance even after the immediate convalescence period following their procedure. When comparing the actual postoperative outcomes to the preoperative baseline, patients made significant improvements in scores for ODI, VAS back, VAS leg, and all ODI subsections except for personal care, which was not significantly different from baseline. This finding further supports the idea that the patients' anticipation of continuing to need assistance with activities of daily living and aspects of self-care following surgery was affirmed during the short-term follow-up period of our study. Finally, when comparing actual outcomes to patient expectations, patients did not report postoperative outcomes that were better than expected for any of the outcome measures, including scores for ODI, VAS back, VAS leg, and most of the ODI subsections. Patients reported similar outcomes compared to expected values for the ODI subcomponents pain intensity, personal care, lifting, and walking.

Our investigation also revealed the strongest correlation

TABLE 2. Comparison of preoperative, expected, and actual postoperative PROs $(n=101)$

\begin{tabular}{|c|c|c|c|c|c|c|c|c|c|}
\hline & \multirow[b]{2}{*}{ Preop (A) } & \multirow[b]{2}{*}{ Expected (B) } & \multirow[b]{2}{*}{ Actual (C) } & \multicolumn{2}{|c|}{$A-B$} & \multicolumn{2}{|c|}{$A-C$} & \multicolumn{2}{|c|}{$B-C$} \\
\hline & & & & $\Delta$ & $\% \Delta$ & $\Delta$ & $\% \Delta$ & $\Delta$ & $\% \Delta$ \\
\hline ODI score & $39.2 \pm 15.7$ & $16.5 \pm 16.7$ & $23.5 \pm 17.4$ & 22.7 & $57.9 \%$ & 15.7 & $40.1 \%$ & -7.0 & $42.4 \%$ \\
\hline VAS back pain score & $5.9 \pm 2.4$ & $2.0 \pm 2.3$ & $3.1 \pm 2.6$ & 3.9 & $66.1 \%$ & 2.8 & $47.5 \%$ & -1.1 & $55 \%$ \\
\hline VAS leg pain score & $5.2 \pm 3.1$ & $1.1 \pm 2.0$ & $2.4 \pm 2.9$ & 4.1 & $78.8 \%$ & 2.8 & $53.8 \%$ & -1.3 & $118.2 \%$ \\
\hline
\end{tabular}

$\Delta=$ difference in absolute values; $\% \Delta=$ percent change.

Mean values are presented \pm SD. 
TABLE 3. Statistical analysis of preoperative, expected, and actual postoperative PROs $(n=101)$

\begin{tabular}{lcccccc}
\hline & & & & \multicolumn{3}{c}{ p Value* } \\
\cline { 5 - 6 } & Preop $(\mathrm{A})$ & Expected $(\mathrm{B})$ & Actual $(\mathrm{C})$ & $\mathrm{A}-\mathrm{B}$ & $\mathrm{A}-\mathrm{C}$ & $\mathrm{B}-\mathrm{C}$ \\
\hline ODI score & $39.2 \pm 15.7$ & $16.5 \pm 16.7$ & $23.5 \pm 17.4$ & $<0.001$ & $<0.001$ & $<0.001$ \\
\hline ODI component score & & & & & \\
\hline Pain intensity & $2.5 \pm 0.9$ & $1.5 \pm 1.3$ & $1.3 \pm 1.1$ & $<0.001$ & $<0.001$ & 0.117 \\
\hline Personal care & $0.7 \pm 0.8$ & $0.9 \pm 0.9$ & $0.8 \pm 1.0$ & $\mathbf{0 . 0 4 1}$ & 0.472 & 0.446 \\
\hline Lifting & $2.7 \pm 1.4$ & $1.8 \pm 1.8$ & $1.9 \pm 1.4$ & $<0.001$ & $<0.001$ & 0.417 \\
\hline Walking & $1.7 \pm 1.1$ & $0.6 \pm 1.1$ & $0.7 \pm 0.9$ & $<0.001$ & $<0.001$ & 0.252 \\
\hline Sitting & $1.9 \pm 1.0$ & $0.7 \pm 1.1$ & $1.3 \pm 1.0$ & $<0.001$ & $<0.001$ & $<0.001$ \\
\hline Standing & $2.6 \pm 1.2$ & $0.7 \pm 1.3$ & $1.4 \pm 1.2$ & $<0.001$ & $<0.001$ & $<0.001$ \\
\hline Sleeping & $1.7 \pm 1.0$ & $0.6 \pm 0.9$ & $1.0 \pm 1.0$ & $<0.001$ & $<0.001$ & $<0.001$ \\
\hline Sex life & $2.0 \pm 1.6$ & $0.5 \pm 1.0$ & $1.2 \pm 1.6$ & $<0.001$ & $<0.001$ & $<0.001$ \\
\hline Social life & $2.2 \pm 1.3$ & $0.6 \pm 1.2$ & $1.1 \pm 1.3$ & $<0.001$ & $<0.001$ & $<0.001$ \\
\hline Traveling & $1.7 \pm 1.0$ & $0.4 \pm 0.9$ & $1.0 \pm 1.0$ & $<0.001$ & $<0.001$ & $<0.001$ \\
\hline VAS back pain score & $5.9 \pm 2.4$ & $2.0 \pm 2.3$ & $3.1 \pm 2.6$ & $<0.001$ & $<0.001$ & $<0.001$ \\
\hline VAS leg pain score & $5.2 \pm 3.1$ & $1.1 \pm 2.0$ & $2.4 \pm 2.9$ & $<0.001$ & $<0.001$ & $<0.001$ \\
\hline
\end{tabular}

Mean values are presented \pm SD. Boldface type indicates statistical significance.

* Calculated using a paired t-test.

between the actual postoperative outcome and patient satisfaction. Better clinical outcomes, as indicated by lower disability and pain scores, were strongly associated with higher postoperative satisfaction reported by patients. Next, the expectation-actuality discrepancy takes into account the closeness of the expected outcome to that actual postoperative outcome. While greater discrepancy was related to poorer patient satisfaction, this was a moderate correlation. Preoperative expectation also demonstrated weak to moderate correlation for all outcome measures. In addition, we determined there to be a moderate correlation between the postoperative change experienced by patients and their postoperative satisfaction for ODI and VAS leg pain, but a strong correlation for VAS back pain. Interestingly, these results reveal that only the actual postopera- tive outcome is strongly correlated with patient satisfaction for all 3 outcome measures. The present study contrasts with past investigations in the available scientific literature that reported a direct relationship between preoperative expectation and postoperative satisfaction. For instance, Cobo Soriano et al. identified optimistic expectations for improvement as a preoperative predictor of significantly improved clinical outcomes following instrumented spinal fusion surgery. ${ }^{5}$ On the other hand, Culliton et al. found that preoperative expectations did not correlate with postoperative satisfaction in patients undergoing primary total knee arthroplasty. ${ }^{6}$ While related investigations have demonstrated mixed results, to our knowledge, our study is the first to demonstrate not only a clear association between actuality and satisfaction, but also the lack of a strong cor-

TABLE 4. Comparison of expected PROs and actual outcomes

Outcome

\begin{tabular}{ll}
\hline \multicolumn{1}{c}{ Outcome } \\
\hline $\begin{array}{ll}\text { Patient expectation compared to preop baseline } \\
\text { Better }\end{array}$ & $\begin{array}{c}\text { ODI; VAS back; VAS leg; ODI sections_-pain intensity, lifting, walking, sitting, standing, sleeping, } \\
\text { sex life, social life, traveling }\end{array}$ \\
\hline Similar & None \\
\hline Worse & ODI personal care \\
\hline Actuality compared to preop baseline & ODI; VAS back; VAS leg; ODI sections-pain intensity, lifting, walking, sitting, standing, sleeping, \\
\hline Better & sex life, social life, traveling \\
\hline Similar & ODI personal care \\
\hline Worse & None \\
\hline Actuality compared to patient expectation & None \\
\hline Better & ODI sections-pain intensity, personal care, lifting, walking \\
\hline Similar & ODI; VAS back; VAS leg; ODI sections-sitting, standing, sleeping, sex life, social life, traveling \\
\hline Worse &
\end{tabular}


TABLE 5. Correlations of expected PROs and postoperative patient satisfaction

\begin{tabular}{|c|c|c|c|c|}
\hline & $\begin{array}{l}\text { Postop Change vs } \\
\text { Satisfaction (r) }\end{array}$ & $\begin{array}{l}\text { Expectation vs } \\
\text { Satisfaction (r) }\end{array}$ & $\begin{array}{l}\text { Expectation-Actuality } \\
\text { Discrepancy vs } \\
\text { Satisfaction (r) }\end{array}$ & $\begin{array}{l}\text { Actuality vs Satisfaction } \\
\text { (r) }\end{array}$ \\
\hline ODI score & 0.447 & 0.353 & 0.432 & 0.717 \\
\hline VAS back pain score & 0.557 & 0.139 & 0.473 & 0.677 \\
\hline VAS leg pain score & 0.381 & 0.298 & 0.411 & 0.610 \\
\hline
\end{tabular}

relation between the expectation-actuality discrepancy and satisfaction.

An important part of making an informed decision and the consent process before undergoing surgery is an open dialogue between the surgeon and the patient regarding the patient's expectations and the realistic outcome from surgical intervention. Providing education regarding the risks and benefits and establishing appropriate expectations are key to counseling and shaping patients' perspectives on their upcoming surgery. In this context, our observational study is one of the first to establish a strong relationship between patient satisfaction and the actual postoperative outcome itself, regardless of preoperative expectation or the realization of expected results. Moreover, to our knowledge, our investigation is the first to demonstrate these findings in the patient population undergoing MIS lumbar fusion procedures. As the quality of healthcare is increasingly being evaluated through patient reports of satisfaction, it is in the best interests of both the patient and the spine surgeon to be cognizant of factors that can influence these measures. With this in mind, our study emphasizes the importance of keeping the focus on achieving the most successful outcomes from surgery regardless of the expectations or mindset that patients may have before undergoing surgery.

Our study is not without limitations. First, the measured outcomes were limited to only one postoperative time point of 6 months. This short-term follow-up may not be indicative of final improvement or outcome, and further analysis of long-term data will better delineate our findings. For instance, differences may be seen at the 12-month time point compared to the 6-month time point. As such, future studies that incorporate a minimum of 12 months of postoperative outcomes will be necessary to better assess patient satisfaction and overall outcome. Second, the study population was not heterogeneous, with the majority of the patients being Caucasian and having private insurance, which may limit the generalizability of the study to a larger, more diverse population. Third, global health outcome measures such as the 12-Item Short-Form Health Survey were not administered. Fourth, factors that could potentially influence postoperative satisfaction independent of outcomes, such as depression/anxiety, narcotics consumption, sex, hospital length of stay, and emergency room visits, were not taken into account or controlled for in our analysis. Fifth, all patients in our study were treated by a single surgeon at a single academic center, which inherently limits the generalizability of the results. Sixth, the standard deviation for expectations was greater than the actual value for the PROs, meaning there was considerable variability in expectations relative to the preoperative PROs and postoperative actual PROs. Last, we did not take into account the different surgical approaches used for the lumbar fusion procedure and their potential influence on

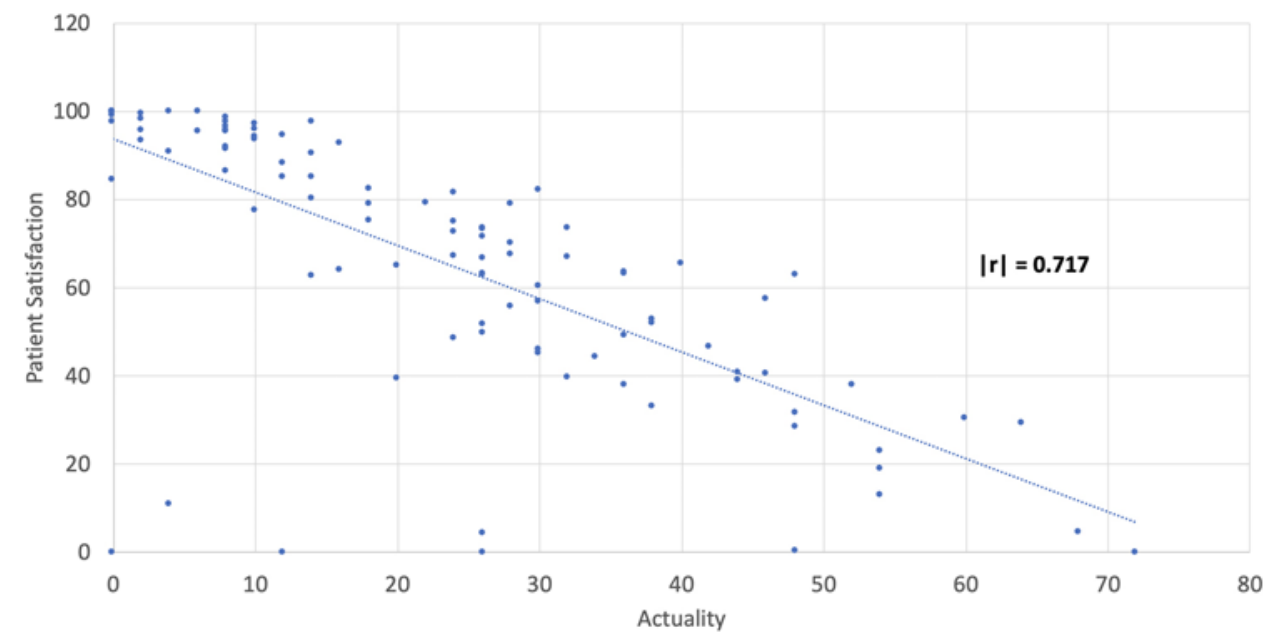

FIG. 1. Patient satisfaction compared to actual postoperative outcomes based on ODI score. Figure is available in color online only. 


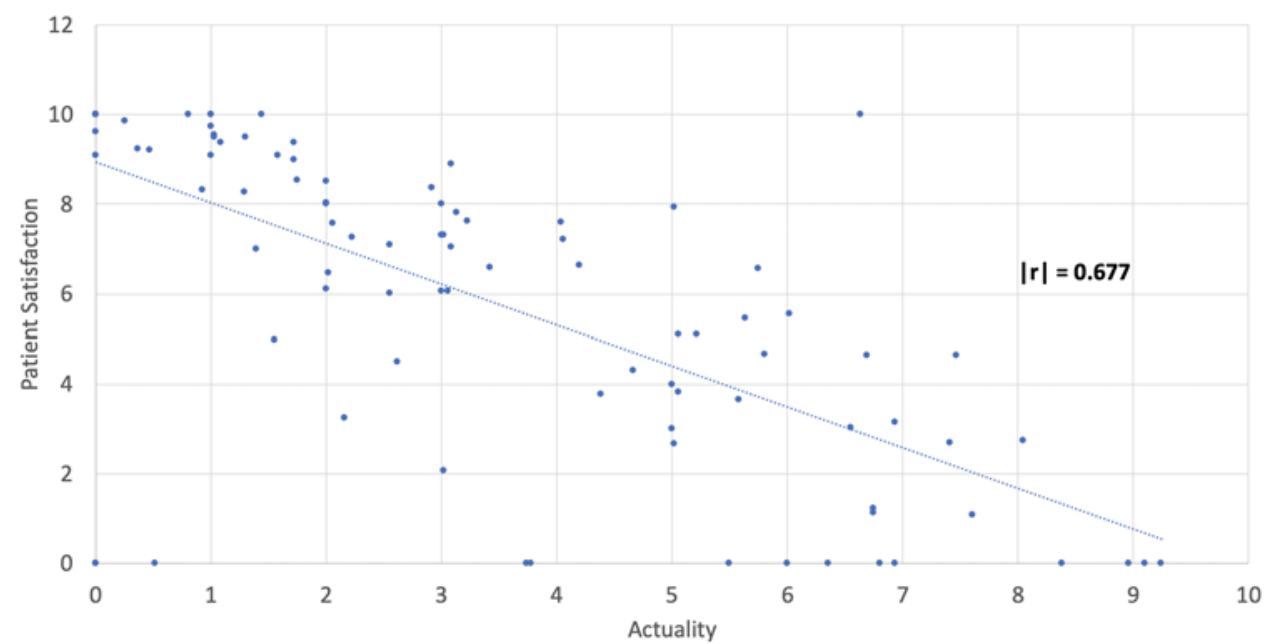

FIG. 2. Patient satisfaction compared to actual postoperative outcomes based on VAS back pain score. Figure is available in color online only.

outcomes. Moving forward, a higher-powered, randomized controlled trial can be conducted to determine the impact of preoperative educational intervention on patient expectations and subsequent postoperative satisfaction. Interventions such as an educational video of the senior surgeon explaining the typical postoperative recovery course may be helpful in further elucidating the influence of preoperative mentality on postoperative outcomes and satisfaction.

\section{Conclusions}

In this observational study, we explored the influence of preoperative expectations and the actual postoperative outcomes, as well the differences between expectations and actual outcomes, with respect to the postoperative satisfaction reported by patients. Interestingly, the actual postoperative results following surgery were strongly correlated with patient satisfaction, while the expectation-actuality discrepancy and the postoperative improvement did not demonstrate strong correlations for all patient-reported outcome measures utilized in our study. Our investigation suggests that the most important indicator of how satisfied patients feel following surgery may be the actual outcome itself, rather than the preoperative expectation or the degree to which the expected result was met. Moving forward, additional studies are warranted to determine the impact of preoperative expectations on patient satisfaction after MIS lumbar fusion spine surgery.

\section{References}

1. Abbott AD, Tyni-Lenné R, Hedlund R: Leg pain and psychological variables predict outcome $2-3$ years after lumbar fusion surgery. Eur Spine J 20:1626-1634, 2011

2. Beecher HK: The powerful placebo. J Am Med Assoc 159:1602-1606, 1955

3. Brook RH: Can the Patient-Centered Outcomes Research Institute become relevant to controlling medical costs and improving value? JAMA 306:2020-2021, 2011

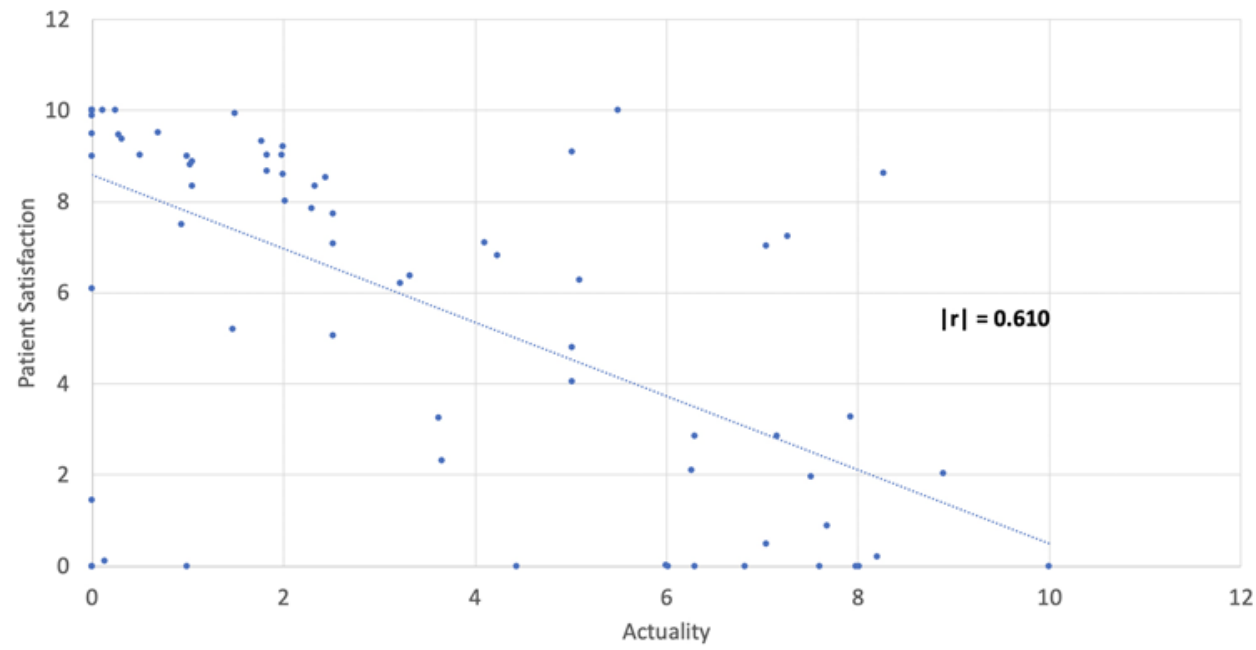

FIG. 3. Patient satisfaction compared to actual postoperative outcomes based on VAS leg pain score. Figure is available in color online only. 
4. Carr FA, Healy KM, Villavicencio AT, Nelson EL, Mason A, Burneikiene S, et al: Effect on clinical outcomes of patient pain expectancies and preoperative Mental Component Summary scores from the 36-Item Short Form Health Survey following anterior cervical discectomy and fusion. J Neurosurg Spine 15:486-490, 2011

5. Cobo Soriano J, Sendino Revuelta M, Fabregate Fuente M, Cimarra Díaz I, Martínez Ureña P, Deglané Meneses R: Predictors of outcome after decompressive lumbar surgery and instrumented posterolateral fusion. Eur Spine J 19:18411848,2010

6. Culliton SE, Bryant DM, Overend TJ, MacDonald SJ, Chesworth BM: The relationship between expectations and satisfaction in patients undergoing primary total knee arthroplasty. J Arthroplasty 27:490-492, 2012

7. de Groot KI, Boeke S, Passchier J: Preoperative expectations of pain and recovery in relation to postoperative disappointment in patients undergoing lumbar surgery. Med Care 37:149-156, 1999

8. den Boer JJ, Oostendorp RA, Beems T, Munneke M, Evers AW: Continued disability and pain after lumbar disc surgery: the role of cognitive-behavioral factors. Pain 123:45-52, 2006

9. Gepstein R, Arinzon Z, Adunsky A, Folman Y: Decompression surgery for lumbar spinal stenosis in the elderly: preoperative expectations and postoperative satisfaction. Spinal Cord 44:427-431, 2006

10. Iversen MD, Daltroy LH, Fossel AH, Katz JN: The prognostic importance of patient pre-operative expectations of surgery for lumbar spinal stenosis. Patient Educ Couns 34:169-178, 1998

11. Lutz GK, Butzlaff ME, Atlas SJ, Keller RB, Singer DE, Deyo RA: The relation between expectations and outcomes in surgery for sciatica. J Gen Intern Med 14:740-744, 1999

12. McGregor AH, Doré CJ, Morris TP: An exploration of patients' expectation of and satisfaction with surgical outcome. Eur Spine J 22:2836-2844, 2013

13. Rönnberg K, Lind B, Zoëga B, Halldin K, Gellerstedt M, Brisby H: Patients' satisfaction with provided care/information and expectations on clinical outcome after lumbar disc herniation surgery. Spine (Phila Pa 1976) 32:256-261, 2007

14. Saban KL, Penckofer SM: Patient expectations of quality of life following lumbar spinal surgery. J Neurosci Nurs 39:180-189, 2007

15. Soroceanu A, Ching A, Abdu W, McGuire K: Relationship between preoperative expectations, satisfaction, and functional outcomes in patients undergoing lumbar and cervical spine surgery: a multicenter study. Spine (Phila Pa 1976) 37:E103-E108, 2012

16. Toyone T, Tanaka T, Kato D, Kaneyama R, Otsuka M: Patients' expectations and satisfaction in lumbar spine surgery. Spine (Phila Pa 1976) 30:2689-2694, 2005
17. Voutilainen A, Pitkäaho T, Kvist T, Vehviläinen-Julkunen K: How to ask about patient satisfaction? The visual analogue scale is less vulnerable to confounding factors and ceiling effect than a symmetric Likert scale. J Adv Nurs 72:946-957, 2016

18. Xiong DD, Ye W, Xiao R, Miller JA, Mroz TE, Steinmetz $\mathrm{MP}$, et al: Patient-reported allergies predict postoperative outcomes and psychosomatic markers after spine surgery. Spine J 19:121-130, 2019

19. Yee A, Adjei N, Do J, Ford M, Finkelstein J: Do patient expectations of spinal surgery relate to functional outcome? Clin Orthop Relat Res 466:1154-1161, 2008

\section{Disclosures}

Dr. Singh reports being a consultant for Zimmer Biomet and K2M; board membership in Vital 5 LLC, TDi LLC, and the Minimally Invasive Spine Study Group; membership on the editorial board for Contemporary Spine Surgery, Orthopedics Today, and Vertebral Columns; receiving royalties from Zimmer Biomet, Stryker, RTI Surgical, Lippincott Williams \& Wilkins, Thieme, Jaypee Publishing, and Slack Publishing; and receiving grants from the Cervical Spine Research Society.

\section{Author Contributions}

Conception and design: Yoo, Patel, Mayo, Massel. Acquisition of data: Karmarkar, Lamoutte. Analysis and interpretation of data: Yoo, Patel, Mayo, Massel. Drafting the article: Yoo, Patel. Critically revising the article: Yoo, Patel, Mayo, Massel. Reviewed submitted version of manuscript: Yoo, Karmarkar. Approved the final version of the manuscript on behalf of all authors: Singh. Statistical analysis: Yoo, Patel. Administrative/technical/material support: Karmarkar, Lamoutte. Study supervision: Singh.

\section{Supplemental Information \\ Online-Only Content}

Supplemental material is available with the online version of the article.

Appendices A and B. https://thejns.org/doi/suppl/10.3171/ 2019.5.SPINE19213.

\section{Correspondence}

Kern Singh: Rush University Medical Center, Chicago, IL. kern. singh@rushortho.com. 